\title{
OSCILAÇÕES CLIMÁTICAS EM MINAS GERAIS
}

\author{
ROSANDRO BOLIGON MINUZZI ${ }^{1}$, RUBENS LEITE VIANELLO², \\ GILBERTO CHOHAKU SEDIYAMA ${ }^{3}$
}

\author{
${ }^{1}$ Universidade Federal de Santa Catarina (UFSC), Florianópolis, SC. \\ ${ }^{2}$ Instituto Nacional de Meteorologia (INMET), Belo Horizonte, MG. \\ ${ }^{3}$ Universidade Federal de Viçosa (UFV), Viçosa, MG.
}

rbminuzzi@cca.ufsc.br; rubens.vianello@inmet.gov.br; g.sediyama@ufv.br

Recebido Dezembro 2007 - Aceito Outubro 2009

\begin{abstract}
RESUMO
O coeficiente angular da análise de regressão e o coeficiente de Mann-Kendall foram utilizados para analisar tendências climáticas mensais da temperatura máxima, da temperatura mínima, da insolação e do conteúdo de vapor d'água, em algumas localidades do Estado de Minas Gerais. Ambos os testes estatísticos foram aplicados para três diferentes períodos, sendo um de 1961 a 2004, e os demais, em intervalos divididos pelo ponto de descontinuidade identificado pelo Teste de Homogeneidade Normal Padrão (THNP). Os resultados mais relevantes indicam que, em Minas Gerais, a temperatura máxima e a temperatura mínima cresceram no período $1960-2004$, entre $1,3^{\circ} \mathrm{C}$ e $3,9^{\circ} \mathrm{C}$, mas este comportamento não foi geralmente gradual, sendo associado com a descontinuidade que ocorreu principalmente na década de 1990. A maior parte do crescimento significativo da temperatura máxima centrou-se entre setembro e dezembro. O predomínio de tendência positiva para o vapor d'água é coerente com o aumento observado dos extremos da temperatura. Entre as poucas tendências significativas da insolação, a predominante foi de decréscimo da década de 1960 até meados da década de 1990.

Palavras-chave: Temperatura, insolação, vapor d'água, clima.
\end{abstract}

ABSTRACT: CLIMATIC OSCILLATIONS IN MINAS GERAIS STATE, BRAZIL

The angular coefficient of the linear regression analysis and the Mann-Kendall's coefficient were used to analyze the monthly climatic trends of the maximum and minimum air temperatures, sunshine and water vapor content, on selected locations in the state of Minas Gerais. Both statistical tests were applied in three different periods, one, from 1961 to 2004, and the other ones at intervals separeted by the discontinuity point detected by the Standard Normal Homogeneity Test (SNHT). The most relevant results indicated that in Minas Gerais the maximum and minimum air temperatures increased in the period $1960-2004$, between $1,3^{\circ} \mathrm{C}$ and $3,9^{\circ} \mathrm{C}$, but this behavior was generally not gradual, being associated with the discontinuity occurring mostly in the 1990's. Most of the significant increase of the maximum temperature was centered between September and December. The dominance of positive trend for water vapor was consistent with the increase in the observed extreme air temperature. It was observed, among the low significant trends of sunshine, a persistence decline from the 1960's until the mid-1990's.

Keywords: Temperature, sunshine, water vapor, climate. 


\section{INTRODUÇÃO}

As várias expressões usadas para descrever as variações no clima, tais como, variabilidade climática, flutuação climática, tendência climática, ciclo climático e mudança climática, referem-se a algumas escalas temporais apropriadas, sendo válidas apenas quando aplicadas em tais escalas. De fato, o clima não é constante e nem sempre foi como hoje o conhecemos. Provas desta afirmativa são os registros de mudanças climáticas globais e, em particular, os regionais, que mostram periodicidades de anos a séculos, durante os quais o clima foi sistematicamente variável.

O tema "mudanças climáticas globais" ganhou ênfase na mídia e despertou o interesse dos governantes em 2007, com o início da divulgação do quarto relatório do Painel Intergovernamental de Mudanças Climáticas (IPCC, na sigla em inglês), exibindo evidências científicas de que o aquecimento global, em grande parte, se deve à ação humana. Artaxo et al. (2003) destacam que as emissões antropogênicas de gases de efeito estufa, ao longo do último século, elevaram as concentrações de $\mathrm{CO}_{2}$ de 280 ppm (partes por milhão), no final do século 19, para os atuais $365 \mathrm{ppm}$, alterando o balanço radiativo e aumentando a temperatura média do ar na superfície terrestre. Outros pesquisadores alertam para a redução da radiação solar incidente à superfície, em decorrência da grande carga de aerossóis na atmosfera. Kaiser e Qian (2002) confirmam tal alerta em estudos realizados na China, com dados de insolação obtidos de 200 estações durante o período de 1954 a 1998. Encontraram uma tendência de decréscimo na insolação de $1,2 \%$ por década que, segundo os autores, pode ser atribuída à rápida atividade de emissões de aerossóis de origem antropogênica, que vêm ocorrendo naquele país nas últimas décadas. Porém, na escala regional estas mudanças são muito divergentes e nem sempre acompanham a tendência global, principalmente em referência a temperatura. Bonsal et al. (2001) analisaram a variabilidade espacial e temporal das temperaturas extremas no Canadá para o período de 1950-1998, e encontraram grandes diferenças na escala regional e sazonal. Chung e Yoon (2000) destacaram que na Coréia entre 1974 a 1997, a temperatura média anual nos grandes centros urbanos aumentou em torno de $1,5^{\circ} \mathrm{C}$, enquanto nas estações marinhas e localizadas na zona rural, os aumentos foram em torno de $0,58^{\circ} \mathrm{C}$. Apesar da grande ressalva que se faz atualmente, quanto a inclusão do fator antropogênico na variabilidade temporal e espacial de elementos meteorológicos, há os fatores naturais resultantes da interação oceano-atmosfera como o El Niño Oscilação Sul (El Niño Southern Oscillation - ENSO), a Oscilação Decadal do Pacífico (Pacific Decadal Oscillation - PDO) e a Oscilação do Atlântico Norte (North Atlantic Oscillation - NAO), além de outras oscilações climáticas naturais, que resultam em flutuações interanuais e decadais em algumas regiões do globo. Eshel (2003) citou alguns estudos como de Hurrel (1995), Johansson et al. (1998), Eshel e Ferrel (2000) e Eshel et al (2000), que apontaram as regiões mais afetadas pela NAO, como sendo o Mediterrâneo e a Europa, principalmente nas variáveis temperatura e precipitação. Particularmente no Brasil, as influências das oscilações climáticas naturais em escala interdecadal ainda são pouco conhecidas. Entre os estudos realizados, o de Teixeira (2002) mostra que em apenas $47 \%$ dos anos analisados, houve concordância entre o sinal da NAO e a qualidade da quadra chuvosa no Ceará, e nem sempre a fase do Dipolo do Atlântico correspondeu ao índice NAO.

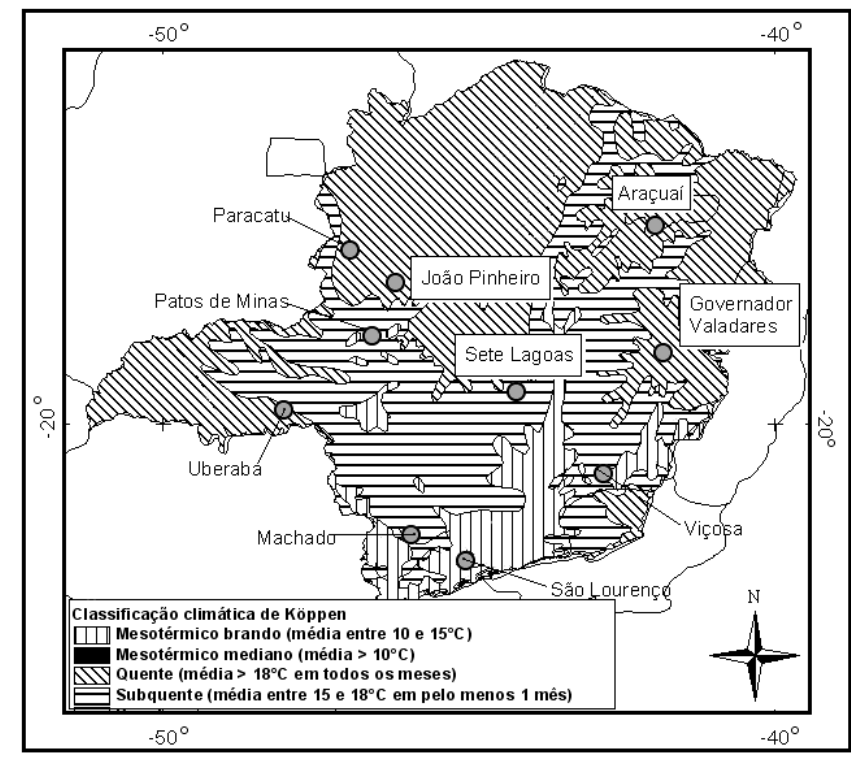

Figura 1 - Localização geográfica das estações meteorológicas estudadas em Minas Gerais. 
No entanto, no Brasil já há indícios de mudanças no comportamento da temperatura do ar, como no estudo de Duursma (2002), citado por Marengo (2006), onde foi encontrado um aumento de $0,6^{\circ} \mathrm{C}$ nos últimos 100 anos, em São Paulo. Contudo, diante da extensão territorial e da diversidade ambiental, há carência de estudos sobre o comportamento dos climas passados no Brasil, prejudicando, inclusive, a alimentação dos modelos de projeções climáticas.

Diante destas questões, este estudo pretende analisar o comportamento mensal da temperatura máxima, temperatura mínima, insolação e do conteúdo de vapor d'água no ar em Minas Gerais, no período de 1961 a 2004 para: 1) identificar pontos de mudança no comportamento climático durante $o$ período de estudo; e 2) determinar as tendências climáticas nestes períodos.

\section{DADOS E METODOLOGIA}

Foram utilizados dados mensais de insolação, umidade do ar, temperatura máxima e temperatura mínima, do período de 1961 a 2004, de dez estações meteorológicas localizadas no Estado de Minas Gerais (Figura 1), pertencentes ao Instituto Nacional de Meteorologia, INMET.

As lacunas de observações foram preenchidas com dados de estações meteorológicas vizinhas, valendo-se do método de regressão linear, considerando $r^{2} \geq 0,7$ como indicador de condições climáticas semelhantes e homogeneidade suficiente para se efetuar o preenchimento. $\mathrm{O}$ teste não-paramétrico denominado 'run test', ou teste de seqüência, foi aplicado para avaliar a homogeneidade das séries meteorológicas, como forma de minimizar o efeito de possíveis fatores não naturais nos registros meteorológicos (erros de leitura, mudança de instrumentos, etc). Recomendado pela Organização Meteorológica Mundial (OMM), em sua Nota Técnica, $n^{\circ}$ 81, o referido teste consiste na contagem do número de ocorrências de valores observados da série temporal, situados abaixo e acima da mediana, testando o resultado da contagem mediante uso de uma tabela de distribuição. Para a pressão de vapor d'água, que é estimada a partir das temperaturas máxima e mínima do ar, foi descartada a série mensal quando estas variáveis foram consideradas não homogêneas.

Dois métodos foram utilizados para testar a tendência das variáveis meteorológicas, a saber, o teste da Análise de Regressão e teste de Mann-Kendall. A Análise de Regressão foi utilizada para indicar alterações climáticas por meio da significância do coeficiente angular de uma reta ajustada aos dados. O teste consiste em determinar o intervalo de confiança do coeficiente, sendo que, se este intervalo não incluir o valor zero, a tendência é significativa. O teste de Mann-Kendall, proposto inicialmente por Sneyers (1975), considera que na hipótese de estabilidade de uma série temporal, a sucessão de valores ocorre de forma independente e a distribuição de probabilidade deve permanecer sempre a mesma (série aleatória simples). Um valor positivo do coeficiente de Mann-Kendall indica uma tendência de aumento da variável, enquanto um valor negativo indica uma tendência de decréscimo, desde que significativos ao nível de 5\%. Ambos os testes foram aplicados para três períodos, sendo que, para a identificação do ponto de descontinuidade das séries temporais foi utilizado o Teste de Homogeneidade Normal Padrão (THNP). Proposto por Alexandersson (1986), o THNP testa um desvio na média $(\bar{Y})$ e estabelece como hipótese nula que a variável mensal $Y_{i}(i$ é o ano de 1 a $\mathbf{n}$ ) a ser testada é independente e identicamente distribuída. Assim, o teste T(k) compara a média dos primeiros $\mathbf{k}$ anos de registros mensais com os últimos $\mathbf{n}-\mathbf{k}$ anos:

$$
\mathrm{T}(\mathrm{k})=\mathrm{k} \overline{\mathbf{z}}_{1}^{2}+(\mathrm{n}-\mathrm{k}) \overline{\mathbf{z}}_{2}^{2} \quad \mathrm{k}=\mathbf{1}, \ldots, \mathrm{n}
$$

onde:

$$
\bar{z}_{1}=\frac{1}{k} \sum_{i=1}^{k} \frac{\left(Y_{i}-\bar{Y}\right)}{s} \quad \text { e } \quad \bar{z}_{2}=\frac{1}{n-k} \sum_{i=k+1}^{n} \frac{\left(Y_{i}-\bar{Y}\right)}{s}
$$

Se o ponto de mudança esta localizado no ano $\mathbf{K}$, então $\mathbf{T}(\mathbf{k})$ atinge um máximo próximo ao ano $\mathbf{k}=\mathbf{K}$. O teste estatístico $\mathbf{T}_{\mathbf{0}}$ é definido como:

$$
T_{0}=\max _{1 \leq k<n} T(k)
$$

A hipótese nula será rejeitada se $\mathbf{T}_{\mathbf{0}}$ estiver acima de um determinado nível, que é dependente do tamanho da amostra. Os valores críticos a $5 \%$ para o teste estatístico de $\mathbf{T}_{\mathbf{0}}$ adotados neste estudo foram definidos por Alexandersson e Moberg (1997).

\section{RESULTADOS E DISCUSSÃO}

A Tabela 1 mostra os anos em que houve descontinuidade no comportamento das séries da temperatura máxima (1a), temperatura mínima (1b), pressão de vapor d'água (1c) e insolação (1d), para localidades e meses em que os dados foram suficientes e homogêneos. Apesar das poucas situações de pontos de descontinuidade estatisticamente significativos a 5\%, em uma análise geral, percebe-se que as mudanças dos extremos da temperatura do ar ocorreram na década de 1990, principalmente, no início (1992) e no final deste período (entre 1997 e 2000). São Lourenço e Viçosa são as únicas localidades que não seguem rigorosamente este argumento para a temperatura máxima e temperatura mínima, respectivamente. Para Viçosa, os pontos de descontinuidade da temperatura mínima ocorreram entre o final da década de 1970 e início da década de 1980 e, ou, não 
houve uma mudança no comportamento, pelo ponto identificado ter sido o primeiro da série temporal (Tabela 1b).

Os resultados da pressão de vapor d'água (Tabela 1c) não seguem rigorosamente os apresentados pelos extremos da temperatura do ar. No entanto, os pontos de descontinuidade foram melhor caracterizados, como em Machado, sendo no início da década de 1990 com seis valores significativos a 5\%.

Para a insolação, apenas ressalta-se os dois únicos pontos de descontinuidade significativos encontrados em Viçosa nos meses de abril e novembro (Tabela 1d), que podem estar associados com o comportamento da temperatura máxima.

A Tabela 2 mostra os coeficientes angulares significativos a 5\%, durante o período de 1961 a 2004 (Tabela 2a) e para dois períodos (Tabela $2 \mathrm{~b}$ e $2 \mathrm{c}$ ) divididos pelo ponto de descontinuidade destacado para a temperatura máxima (Tabela 1a). A tendência foi considerada significativa quando se verificou consenso entre os sinais obtidos pela análise de regressão e o coeficiente de Mann-Kendal, desde que, atestado a significância de ambos. Foram observadas somente crescimentos de 1961 a 2004, com aumentos nos últimos 44 anos de $1,3^{\circ} \mathrm{C}$ a $3,9^{\circ} \mathrm{C}$. Este resultado deixa claro que há pontos de descontinuidade significativa nas séries, provavelmente devido à mudanças de fase de oscilações interdecadais, mas não há tendências consistentes nos períodos separados por estes pontos. Os crescimentos entre 1961 e 2004 foram maiores na região compreendida pelos municípios de João Pinheiro, Paracatu e Patos de Minas, notadamente durante a primavera (setembro a novembro), como em Paracatu, que apresentou a partir de 1993, apenas um registro abaixo da média climática do período para o mês de setembro (Figura 2). Outubro e dezembro mostraram serem os meses com maior número

Tabela 1 - Pontos de descontinuidade no comportamento climático da temperatura máxima (1a), da temperatura mínima (1b), da pressão do vapor d'água (1c) e da insolação (1d), para os municípios de Machado (MAC), São Lourenço (SLOU), Viçosa (VIC), Sete Lagoas (SETE), João Pinheiro (JPIN), Paracatu (PAR), Patos de Minas (PAT), Uberaba (UBE), Araçuaí (ARAÇ) e Governador Valadares (GOV)

\begin{tabular}{|c|c|c|c|c|c|c|c|c|}
\hline \multirow[t]{13}{*}{$1 a$} & & MAC & SLOU & VIÇ & JPIN & PAR & PAT & UBE \\
\hline & Jan & 1997 & 1 & 1961 & 961 & 1 & 1961 & 1961 \\
\hline & $\mathrm{Fev}$ & 1 & 1983 & 1993 & 993 & 1 & 1993 & 1972 \\
\hline & Mar & 1997 & 1976 & 2000 & $2000 *$ & 2000 & 2 & 1990 \\
\hline & Abr & $2000 *$ & 1 & $2000 *$ & $000 \quad 2000 *$ & 1 & 2 & $1997 *$ \\
\hline & Mai & 1999 & 1983 & 2000 & 1 & 1 & 1 & 1985 \\
\hline & Jun 1 & 1999* & 2 & 1 & 1 & 1 & 1 & 1997 \\
\hline & Jul & 2000 & 1992 & 1964 & 1992 & $1997^{*}$ & 2 & 1997 \\
\hline & Ago & 1994 & 1993 & 1994 & 1992 & 1 & 1968 & 1993 \\
\hline & Set & 1 & 1961 & 2001 & $961 \quad 1992 *$ & $1992 *$ & 1992 & 1961 \\
\hline & Out & 1999 & 1983 & 1999 & 992 2001* & $1992 *$ & 1992 & 1 \\
\hline & Nov & 1981 & 1 & $961 *$ & 997 2001* & 2001* & 2 & 1963 \\
\hline & Dez 1 & 1996* & 1986 & 1995 & 993 & 1 & 2 & 2001 \\
\hline & MAC & VIC & UBE & GV & & $1 \mathrm{c}$ & & MAC \\
\hline Jan & 1993 & $1997 *$ & 1 & 1 & & & Jan & 1993* \\
\hline Fev & 1 & 1982 & 1 & 1 & & & Fev & 1 \\
\hline Mar & 1993 & 1 & 1997 & 1994 & & & Mar & $1993^{*}$ \\
\hline Abr & 1 & 1 & 2000 & 2000 & & & Abr & $1991^{*}$ \\
\hline Mai & 1978 & 1978 & 1968 & 2000 & & & Mai & 1991 \\
\hline Jun & 1962 & 1979 & 1 & 1 & & & Jun & 1991 \\
\hline Jul & 1986 & 1962 & 1997 & 1998 & & & Jul & $1991^{*}$ \\
\hline Ago & 2003 & 1961 & 1997 & 2000 & & & Ago & 1991 \\
\hline Set & 1991 & 1982 & 2003 & 1 & & & Set & 1991 \\
\hline Out & 1 & 1961 & 1 & 2001 & & & Out & $1991^{*}$ \\
\hline Nov & 1991 & $1961^{*}$ & 1999 & 2002 & & & Nov & 1992 \\
\hline \multirow[t]{14}{*}{ Dez } & 1992 & 1 & 1 & 1 & & & Dez & $1992 *$ \\
\hline & & & $1 d$ & MAC & VIÇ & SETE & UBE & GV \\
\hline & & & Jan & 1 & 1978 & 1961 & 1981 & 2 \\
\hline & & & Fev & 1 & 2001 & 1 & 1 & 1968 \\
\hline & & & Mar & 1 & 2000 & 1990 & 1990 & 2003 \\
\hline & & & Abr & 1998 & $2000^{*}$ & 2 & 1961 & 1976 \\
\hline & & & Mai & 1975 & 1998 & 1 & 1 & 1975 \\
\hline & & & Jun & 1978 & 1961 & 2 & 1999 & 1 \\
\hline & & & Jul & 1987 & 1964 & 2 & 1968 & 1 \\
\hline & & & Ago & 1983 & 1 & 1983 & 1975 & 1996 \\
\hline & & & Set & 1975 & 1961 & 2 & 2003 & 1 \\
\hline & & & Out & 1 & 1999 & 1992 & 1 & 1 \\
\hline & & & Nov & 1974 & $1961^{*}$ & 1999 & 1993 & 1989 \\
\hline & & & Dez & 1975 & 2000 & 1 & 1962 & 1 \\
\hline
\end{tabular}

\footnotetext{
${ }^{1}$ Série de dados não homogêneas ou ambas as séries dos extremos da temperatura do ar não homogêneas (referência à pressão de vapor d'água)

${ }^{2}$ Número de dados insuficientes para análise

* Significativo ao nível de 5\%.
} 
de tendências significativas em 1961-2004 em Minas Gerais, enquanto Araçuaí e Uberaba foram os municípios com menores números de tendências significativas em 1961-2004 (Tabela 2a). Uma explicação pode estar no comportamento decrescente observado em períodos menores, como em Araçuaí no mês de abril, de 1961 a 1985 (Tabela 2b) e em janeiro, de 1994 a 2004 (Tabela 2c); e em Uberaba, uma tendência positiva observada em março, mas de forma significativa somente a partir da década de 1990 (Tabela 2c).

À semelhança da temperatura máxima, a mínima também mostrou crescimento entre 1961 a 2004, com destaque para o município de Viçosa, com aumento de $0,86^{\circ} \mathrm{C}$ a $3,4^{\circ} \mathrm{C}$ em sete dos nove meses analisados (Tabela 3a). Dentre estes meses, em janeiro e setembro a tendência foi significativa até 1997 e até 1982, respectivamente (Tabela 3b). Nas demais localidades analisadas, praticamente não houve tendência positiva significativa no comportamento da temperatura mínima (Tabela 3b e 3c). Uberaba e Governador Valadares foram as que apresentaram menor tendência positiva na temperatura mínima.

A Figura 3 apresenta os valores observados da temperatura mínima para janeiro em Viçosa, que mostrou

Tabela 2 - Coeficientes angulares da temperatura máxima de 1961 a 2004 (2a) e para dois períodos (Tabela 2b e 2c) divididos pelo ponto de mudança climática, nos municípios de Machado (MAC), São Lourenço (SLOU), Viçosa (VIÇ), Sete Lagoas (SETE), João Pinheiro (JPIN), Paracatu (PAR), Patos de Minas (PAT), Uberaba (UBE) e Araçuaí (ARAÇ)

\begin{tabular}{lccccccccc}
\cline { 2 - 10 } 2a & MAC & SLOU & VIÇ & SETE & JPIN & PAR & PAT & UBE & ARAÇ \\
\hline Jan & 0 & 1 & 0 & 0 & 1 & 1 & 0 & 0 & 0 \\
Fev & 1 & 0,04 & 0,04 & 0,03 & 1 & 1 & 0,03 & 0 & 0 \\
Mar & 0 & 0 & 0 & 2 & 0,04 & 0,04 & 2 & 0 & 0 \\
Abr & 0,04 & 1 & 0,05 & 0 & 0,04 & 1 & 2 & 0 & 0 \\
Mai & 0 & 0 & 0,03 & 2 & 1 & 1 & 1 & 0 & 0 \\
Jun & 0 & 2 & 1 & 2 & 1 & 1 & 1 & 0 & 1 \\
Jul & 0,04 & 0 & 0,03 & 2 & 0,03 & 0,08 & 2 & 0 & 0 \\
Ago & 0 & 0 & 0 & 0 & 0 & 1 & 0 & 0 & 0 \\
Set & 1 & 0 & 0 & 0 & 0,05 & 0,09 & 0,04 & 0 & 0 \\
Out & 0,05 & 0,05 & 0 & 0,05 & 0,08 & 0,08 & 0,05 & 1 & 0,05 \\
Nov & 0,03 & 1 & 0 & 0 & 0,06 & 0,06 & 2 & 0 & 0 \\
Dez & 0,04 & 0,04 & 0,05 & 0,03 & 2 & 1 & 2 & 0 & 0,06 \\
\hline & & & & & & & & &
\end{tabular}

\begin{tabular}{lccccccccc}
\cline { 2 - 9 } b & MAC & SLOU & VIC & SETE & JPIN & PAR & PAT & UBE & ARAC \\
\hline Jan & 0 & 1 & 3 & 3 & 1 & 1 & 3 & 3 & 0 \\
Fev & 1 & 0 & 0 & 0 & 1 & 1 & 0 & 0 & 0 \\
Mar & 0 & 0 & 0 & 2 & 0 & 0 & 2 & 0 & 0 \\
Abr & 0 & 1 & 0 & 0 & 0 & 1 & 2 & 0 & $-0,11$ \\
Mai & 0 & 0 & 0 & 2 & 1 & 1 & 1 & 0 & 0 \\
Jun & 0 & 2 & 1 & 2 & 1 & 1 & 1 & 0 & 1 \\
Jul & 0 & 0 & 0 & 2 & 0 & 0,06 & 2 & 0 & 0 \\
Ago & 0 & 0 & 0 & 0 & 0 & 1 & 0 & $-0,04$ & 0 \\
Set & 1 & 3 & 3 & 3 & 0 & 0 & 0 & 3 & 0 \\
Out & 0 & 0 & 0 & 0 & 0,05 & 0 & 0 & 1 & 0 \\
Nov & 0 & 1 & 3 & 0 & 0 & 0 & 2 & 0 & 0 \\
Dez & 0 & 0 & 0 & 0 & 2 & 1 & 2 & $-0,05$ & 0
\end{tabular}

\begin{tabular}{lccccccccc}
\cline { 2 - 9 } 2c & MAC & SLOU & VIC & SETE & JPIN & PAR & PAT & UBE & ARAC \\
\hline Jan & 0 & 1 & 3 & 3 & 1 & 1 & 3 & 3 & $-0,84$ \\
Fev & 1 & 0 & 0 & 0 & 1 & 1 & 0 & 0 & 0 \\
Mar & 0 & 0 & 0 & 2 & 0 & 0 & 2 & 0,17 & 0 \\
Abr & 0 & 1 & 0 & 0 & 0 & 1 & 2 & 0 & 0 \\
Mai & 0 & $-0,09$ & 0 & 2 & 1 & 1 & 1 & 0 & 0 \\
Jun & 0 & 2 & 1 & 2 & 1 & 1 & 1 & 0 & 1 \\
Jul & 0 & 0 & 0 & 2 & 0 & 0 & 2 & 0 & 0 \\
Ago & 0 & 0 & 0 & 0 & 0 & 1 & 0 & 0 & 0 \\
Set & 1 & 3 & 3 & 3 & 0 & 0 & 0 & 3 & 0 \\
Out & 0 & 0 & 0 & 0 & 0 & 0 & 0 & 1 & 0 \\
Nov & 0 & 1 & 3 & 0 & 0 & 0 & 2 & 0 & 0 \\
Dez & 0 & 0 & 0 & 0 & 2 & 1 & 2 & 0 & 0 \\
\hline
\end{tabular}

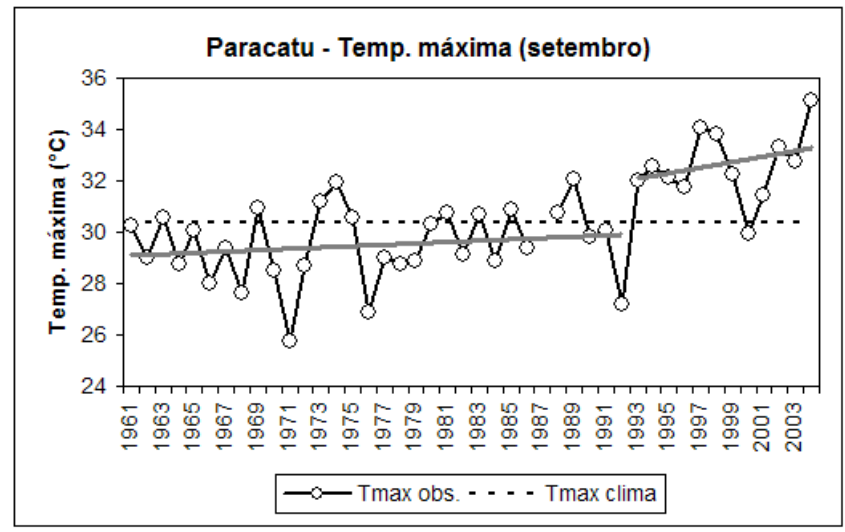

Figura 2 - Comportamento temporal da temperatura máxima em setembro, no município de Paracatu e suas respectivas tendências nos períodos divididos pelo ponto de mudança climática. 
uma tendência gradual de aumento da temperatura no período de 1961 a 1997. A partir deste ano (1997), apesar de todas as temperaturas registradas estarem acima da média climatológica, estas não seguiram a tendência crescente observada entre as décadas de 1960 e 1990.

Sendo uma variável dependente da temperatura do ar, a pressão do vapor também mostrou crescimento nos últimos 44 anos analisados, nas localidades e meses em que os dados foram suficientes e consistentes, exceto no município de Uberaba. Em Viçosa, observou-se aumento na pressão de vapor d'água entre $0,35 \mathrm{mb} /$ década a $0,6 \mathrm{mb} /$ década, de janeiro a junho; em Machado, de 0,54 mb/década a 0,88 mb/década, em dez dos 11 meses analisados (Tabela 4a). Resultados semelhantes de aumento foram encontrados numa região semi-árida da Turquia, com o uso de três diferentes testes não-paramétricos. Tonkaz et al. (2007) encontraram, na maioria das estações analisadas, um

Tabela 3 - Coeficientes angulares da temperatura mínima de 1961 a 2004 (3a) e para dois períodos (Tabela 3b e 3c) divididos pelo ponto de mudança climática, nos municípios de Machado (MAC), Viçosa (VIÇ), Uberaba (UBE) e Governador Valadares (GV)

\begin{tabular}{lcccc}
\cline { 2 - 5 } 3a & MAC & VIC & UBE & GV \\
\hline Jan & 0,02 & 0,04 & 1 & 1 \\
Fev & 1 & 0,03 & 1 & 1 \\
Mar & 0,02 & 1 & 0 & 0 \\
Abr & 1 & 1 & 0 & 0 \\
Mai & 0 & 0,08 & 0 & 0 \\
Jun & 0 & 0,06 & 1 & 1 \\
Jul & 0 & 0,05 & 0 & 0 \\
Ago & 0 & 0 & 0 & 0 \\
Set & 0 & 0,04 & 0 & 1 \\
Out & 1 & 0,02 & 1 & 0 \\
Nov & 0 & 0 & 0,02 & 0 \\
Dez & 0,02 & 1 & 1 & 1 \\
\hline
\end{tabular}

\begin{tabular}{|c|c|c|c|c|}
\hline $3 b$ & MAC & VIC & UBE & GV \\
\hline Jan & 0 & 0,16 & 1 & 1 \\
\hline Fev & 1 & 0 & 1 & 1 \\
\hline Mar & 0 & 1 & 0 & 0 \\
\hline Abr & 1 & 1 & 0 & 0 \\
\hline Mai & 0 & 0 & 0 & 0 \\
\hline Jun & 3 & 0 & 0 & 1 \\
\hline Jul & 0 & 3 & 0 & 0 \\
\hline Ago & 0 & 3 & 0 & 0 \\
\hline Set & 0 & 0,08 & 3 & 1 \\
\hline Out & 1 & 3 & 1 & 0 \\
\hline Nov & 0 & 3 & 0 & 0 \\
\hline Dez & 0 & 1 & 1 & 1 \\
\hline
\end{tabular}

\begin{tabular}{lcccc} 
3c & MAC & VIC & UBE & GV \\
\hline Jan & 0 & 0 & 1 & 1 \\
Fev & 1 & 0 & 1 & 1 \\
Mar & 0 & 1 & $-0,17$ & 0 \\
Abr & 1 & 1 & 0 & 0 \\
Mai & 0 & 0 & 0 & 0 \\
Jun & 3 & 0 & 1 & 1 \\
Jul & 0 & 3 & 0 & 0 \\
Ago & 0 & 3 & 0 & 0 \\
Set & 0 & 0 & 3 & 1 \\
Out & 1 & 3 & 1 & 0 \\
Nov & 0 & 3 & 0 & 0 \\
Dez & 0 & 1 & 1 & 1 \\
\hline
\end{tabular}

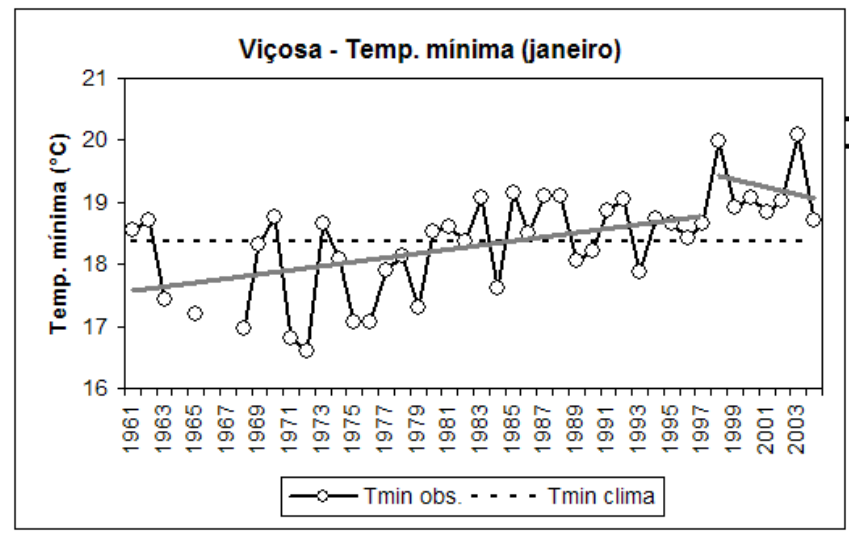

Figura 3 - Comportamento temporal da temperatura mínima em janeiro, no município de Viçosa e suas respectivas tendências nos períodos divididos pelo ponto de mudança climática. 
aumento linear da pressão de vapor, em três diferentes períodos (1978 a 1984, 1984 a 1997 e 1997 a 2002), em especial no verão, seguindo-se o outono. Os pesquisadores atribuíram à construção de barragens, início das práticas de irrigação em grande escala, mudanças nos padrões de cultivo agrícola e aos padrões globais, as principais causas de tais aumentos no vapor d'água. Em escala global, Willet et al. (2007), usando dados obtidos em estações meteorológicas, navios e bóias localizadas em diversos pontos, constataram uma tendência de aumento do vapor d'água de $2,2 \%$, em quase todo o globo (exceto na Austrália e no Oceano Ártico), entre 1976 a 2004, associando essa tendência com o aumento da temperatura. Tal associação entre a umidade do ar e a temperatura tem sido interpretada como prova das interações existentes entre componentes da atmosfera, denominadas "mecanismos de realimentação" (feedback). Bengtsson (2001) sugeriu que um aumento da ordem de $1^{\circ} \mathrm{C}$ na temperatura do ar deve induzir um aumento no conteúdo de umidade em aproximadamente $6 \%$, tornando os eventos de chuvas extremas mais freqüentes. No entanto, tais afirmações não são válidas para o comportamento observado em Uberaba, já que o município do Triângulo Mineiro mostrou dois períodos com comportamentos distintos do vapor d'água, sendo de diminuição a partir de meados da década de 1970 durante os primeiros cinco meses do ano (Tabela 4c) e de aumento nos demais meses, observados até o início da década de 1990 (Tabela 4b), não estando associados com as tendências observadas da temperatura máxima e mínima. A Figura 4 ilustra esta situação utilizando o mês de março como exemplo, que teve

Tabela 4 - Coeficientes angulares da pressão de vapor d'água de 1961 a 2004 (4a) e para dois períodos (Tabela 4b e 4c) divididos pelo ponto de mudança climática, nos municípios de Machado (MAC), Viçosa (VIÇ) e Uberaba (UBE)

\begin{tabular}{lccc}
\cline { 2 - 4 } 4a & MAC & VIC & UBE \\
\hline Jan & 0,08 & 0,04 & 0 \\
Fev & 1 & 0,03 & 1 \\
Mar & 0,08 & 0,05 & 0 \\
Abr & 0,09 & 0,06 & 0 \\
Mai & 0,07 & 0,06 & 0 \\
Jun & 0,06 & 0,04 & 0 \\
Jul & 0,07 & 0 & 0 \\
Ago & 0,05 & 0 & 0 \\
Set & 0,07 & 0,05 & 0 \\
Out & 0 & 0 & 1 \\
Nov & 0,05 & 0 & 0 \\
Dez & 0,07 & 0 & 1 \\
\hline
\end{tabular}

\begin{tabular}{lccc}
\cline { 2 - 4 } 4b & MAC & VIC & UBE \\
\hline Jan & 0 & 0 & 0 \\
Fev & 1 & 0 & 1 \\
Mar & 0 & 0 & 0 \\
Abr & 0 & 0 & 0 \\
Mai & 0 & 0 & 0 \\
Jun & 0 & 0 & 0,28 \\
Jul & 0 & 3 & 0,34 \\
Ago & 0 & 0,23 & 0,23 \\
Set & 0 & 3 & 0,12 \\
Out & $-0,06$ & 3 & 1 \\
Nov & 0 & 0 & 0,19 \\
Dez & 0 & 0 & 1 \\
\hline
\end{tabular}

\begin{tabular}{lccc}
\cline { 2 - 4 } 4c & MAC & VIC & UBE \\
\hline Jan & 0 & 0 & $-0,18$ \\
Fev & 1 & 0 & 1 \\
Mar & 0 & 0 & $-0,18$ \\
Abr & 0 & 0 & $-0,13$ \\
Mai & 0 & 0 & $-0,26$ \\
Jun & 0 & 0 & 0 \\
Jul & 0 & 0 & 0 \\
Ago & 0 & 0 & 0 \\
Set & 0 & 0 & 0 \\
Out & 0 & 0 & 1 \\
Nov & 0 & 0 & 0 \\
Dez & 0 & 0 & 1 \\
\hline
\end{tabular}

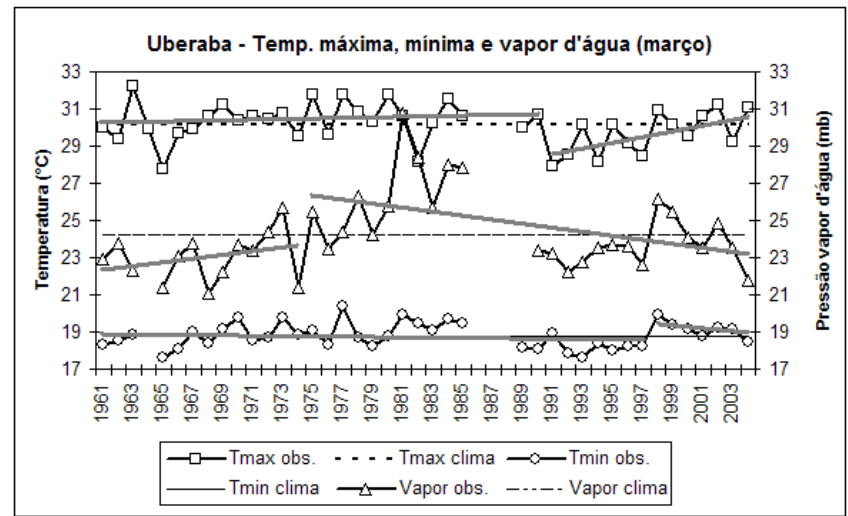

Figura 4 - Comportamento temporal da temperatura máxima, mínima e pressão de vapor d'água em março, no município de Uberaba e suas respectivas tendências nos períodos divididos pelo ponto de mudança climática. 
uma tendência de decréscimo do conteúdo de vapor d'água a partir de 1974, enquanto a temperatura máxima e temperatura mínima tiveram, respectivamente, uma tendência de aumento e diminuição a partir da década de 1990. Ressalte-se que esta tendência pode ser diferente, dependendo dos valores nos anos de dados faltantes.

A insolação foi a variável com o menor número de tendências significativas e de maior oscilação de sinais, em uma mesma estação e entre as estações distintas (Tabela 5). Uberaba, por exemplo, mostrou decréscimo em janeiro e novembro, de 1961 a 2004 (Tabela 5a), e tendências positivas, em agosto, de 1961 a 1975 (Tabela 5b) e em março, a partir de 1990 (Tabela 5c). Os resultados conflitantes podem estar associados ao excessivo número de séries inconsistentes, que impedem conclusões mais objetivas. Em três grandes cidades do leste da China, Zhang et al. (2004) constataram um decréscimo da radiação solar global e direta, atribuindo à aceleração da poluição do ar e à diminuição da insolação como possíveis causas do fenômeno.

Em suma, os resultados evidenciaram aumento das temperaturas máximas e mínimas no período 1961-2004, embora seja perceptível que este comportamento geralmente não é gradual; em raras situações, apresenta em algum intervalo do período analisado (1961 a 2004), tendência significativa de decréscimo. Comportamento semelhante é observado para o vapor d'água em duas das três localidades analisadas. Assim, no geral, os resultados encontrados mostram certa coerência, considerando as relações termodinâmicas entre temperatura e pressão de vapor. Contudo, é necessário ressaltar que geralmente não houve tendência positiva para as temperaturas máximas e mínimas em períodos homogêneos das séries, e que os aumentos observados devem-se aos pontos de descontinuidade nas séries, que podem ser devido às mudanças de fase de oscilações interdecadais naturais.

Se houver participação do fator antropogênico no aumento da temperatura, e conseqüente aumento da pressão de vapor, pode haver um mecanismo de realimentação, ou seja, a maior concentração de poluentes no ar, como o $\mathrm{CO}_{2}$, captaria mais energia, aquecendo mais a atmosfera, aumentando a evaporação e o vapor d'água atmosférico, que captaria mais energia, aquecendo ainda mais a atmosfera (Flannery, 2007). Seguindo esse raciocínio, Easterling et al. (1997) analisaram as tendências nos extremos da temperatura do ar global e a conseqüente implicação na amplitude térmica. Com dados de 5400 estações meteorológicas distribuídas pelo mundo (54\% da superfície terrestre) encontraram uma tendência de aumento da temperatura máxima de $0,88^{\circ} \mathrm{C} /$ século. Com o aumento da

Tabela 5 - Coeficientes angulares da insolação de 1961 a 2004 (5a) e para dois períodos (Tabela 5b e 5c) divididos pelo ponto de mudança climática, nos municípios de Machado (MAC), Viçosa (VIÇ), Sete Lagoas (SETE), Uberaba (UBE) e Governador Valadares (GV)

\begin{tabular}{lccccc}
\cline { 2 - 6 } 5a & MAC & VIC & SETE & UBE & GV \\
\hline Jan & 1 & 0 & 0 & $-0,03$ & 2 \\
Fev & 1 & 0 & 1 & 1 & 2 \\
Mar & 1 & 0 & 0 & 0 & 0 \\
Abr & 0 & 0 & 2 & 0 & 0 \\
Mai & $-0,03$ & 0 & 1 & 1 & 0 \\
Jun & 0 & 0 & 2 & 0 & 1 \\
Jul & $-0,02$ & 0 & 2 & 0 & 1 \\
Ago & 0 & 1 & $-0,03$ & 0 & 0 \\
Set & 0 & 0 & 2 & 0 & 1 \\
Out & 1 & 0 & 0,05 & 1 & 1 \\
Nov & 0 & 0 & 0 & $-0,03$ & 0 \\
Dez & 0 & 0 & 1 & 0 & 1 \\
\hline
\end{tabular}

\begin{tabular}{lccccc}
\cline { 2 - 6 } b & MAC & VIC & SETE & UBE & GV \\
\hline Jan & 1 & 0 & 3 & 0 & 2 \\
Fev & 1 & 0 & 1 & 1 & 2 \\
Mar & 1 & 0 & 0 & 0 & 3 \\
Abr & $-0,04$ & 0 & 2 & 3 & 0 \\
Mai & 0 & 0 & 1 & 1 & 0 \\
Jun & 0 & 3 & 2 & 0 & 1 \\
Jul & 0 & 0 & 2 & 0 & 1 \\
Ago & 0 & 1 & 0 & 0,06 & $-0,05$ \\
Set & $-0,1$ & 3 & 2 & 3 & 1 \\
Out & 1 & 0 & 0 & 1 & 1 \\
Nov & 0 & 3 & 0 & 0 & $-0,11$ \\
Dez & 0 & 0 & 1 & 3 & 1 \\
\hline
\end{tabular}

\begin{tabular}{lccccc}
\cline { 2 - 6 } 5c & MAC & VIC & SETE & UBE & GV \\
\hline Jan & 1 & 0 & 3 & 0 & 2 \\
Fev & 1 & 0 & 1 & 1 & 2 \\
Mar & 1 & 0 & 0 & 0,15 & 3 \\
Abr & 0 & 0 & 2 & 3 & 0 \\
Mai & 0 & 0 & 1 & 1 & 0 \\
Jun & 0 & 3 & 2 & 0 & 1 \\
Jul & 0 & 0 & 2 & 0 & 1 \\
Ago & 0,09 & 1 & 0 & 0 & 0 \\
Set & 0 & 3 & 2 & 3 & 1 \\
Out & 1 & 0 & 0 & 1 & 1 \\
Nov & 0 & 3 & 0 & 0 & 0 \\
Dez & 0 & 0 & 1 & 3 & 1 \\
\hline
\end{tabular}

\footnotetext{
${ }^{1}$ Série de dados não homogêneas ou ambas as séries dos extremos da temperatura do ar não homogêneas (referência à pressão de vapor d'água)

${ }^{2}$ Número de dados insuficientes para análise

${ }^{3}$ Ponto de mudança climática no primeiro/segundo ou penúltimo/último ano da série temporal analisada

0 Sem tendência significativa ao nível de 5\%
} 
temperatura mínima de $1,86^{\circ} \mathrm{C} /$ século, ficou constatado um decréscimo da amplitude. Os resultados foram semelhantes quando analisado o efeito urbano, ou seja, considerando somente estações meteorológicas localizadas em cidades com populações acima de 50 mil (1300 estações). Na América do Sul, para o aumento significativo da temperatura mínima, principalmente a partir da década de 1970, os cientistas apontaram como fator principal o aumento da nebulosidade. No nosso estudo, ao contrário de Easterling et al. (1997), as temperaturas mínimas mostram menos crescimento que as máximas.

Torna-se difícil apontar uma associação dos resultados encontrados em Minas Gerais, com oscilações interdecadais como a PDO e a NAO, considerando os poucos estudos, que indiquem a influência destas forçantes no clima do Brasil e pela diversidade dos pontos de descontinuidade e respectivas tendências climáticas obtidas entre estes pontos.

Os resultados apresentados das quatro variáveis meteorológicas em Uberaba dificultam uma conclusão mais precisa para o comportamento da pressão de vapor, seja em relação a erros nos registros instrumentais ou de mudanças microclimáticas associadas, principalmente, às alterações no ambiente em torno das estações.

Não menos importante é a hipótese de erros observacionais causados por falhas humanas, por manutenção deficiente de equipamentos e por descontinuidades das observações. Ainda que se usem métodos estatísticos para o preenchimento de séries incompletas e que se façam análises de homogeneidade, os dados finais disponíveis tornam-se precários ou insuficientes, impedindo análises mais amplas, consistentes, e resultados mais confiáveis. Por tais razões, inúmeras localidades deixaram de ser analisadas nesta pesquisa. Mesmo com o preenchimento de lacunas nas séries, não houve dados suficientes para realizar análises mensais de temperatura máxima em Governador Valadares; de temperatura mínima em João Pinheiro, Pato de Minas, Paracatu e Sete Lagoas; de insolação em Araçuaí, Patos de Minas, Paracatu e São Lourenço; de pressão de vapor em Araçuaí, Patos de Minas, Paracatu, São Lourenço, Governador Valadares e Sete Lagoas. Assim, percebe-se a necessidade em investir mais na coleta e na qualidade dos dados, para que estudos referentes ao comportamento climático possam ser melhores compreendidos.

\section{CONCLUSÕES}

Para as localidades estudadas de Minas Gerais, pode-se concluir que:

- Apesar dos poucos pontos de descontinuidade no clima estatisticamente significativos, as mudanças no comportamento da temperatura máxima e mínima ocorreram na década de 1990. São Lourenço e Viçosa são as únicas localidades que não seguem rigorosamente este argumento para a temperatura máxima e temperatura mínima, respectivamente.

- Os extremos máximo e mínimo da temperatura do ar mostraram aumento desde a década de 1960, porém, este comportamento geralmente não é gradual, sendo associado com as descontinuidades detectadas, que podem dever-se a mudanças de fase de oscilações interdecadais.

- O maior número de tendências significativas e persistentes da temperatura máxima ficou centrado entre setembro e dezembro, principalmente durante o último mês do ano. Os aumentos desta variável meteorológica, no período de 1961 a 2004 , oscilaram entre $1,3^{\circ} \mathrm{C}$ e $3,9^{\circ} \mathrm{C}$.

- O predomínio de aumento na tendência da quantidade de vapor d'água é coerente com o aumento observado nos extremos máximo e mínimo da temperatura do ar, porém, os pontos de descontinuidade no comportamento do vapor d'água não seguiram rigorosamente os apresentados pelos extremos da temperatura do ar.

- O município de Uberaba foi o que contabilizou o menor número de tendências significativas da temperatura do ar, bem como o único a apresentar tendência de decréscimo no conteúdo de vapor d'água a partir de meados da década de 1970, durante os primeiros cinco meses do ano e de aumento nos demais meses, observados até o início da década de 1990.

- Houve poucas indicações de tendências significativas para a insolação, e dentre estas, a predominante foi de decréscimo da década de 1960 até meados da década de 1990.

\section{REFERÊNCIAS BIBLIOGRÁFICAS}

ALEXANDERSSON, H. A homogeneity test applied to precipitation data. Journal of Climatology, v.6, p.661-675, 1986.

ALEXANDERSSON, H.; MOBERG, A. Homogenization of Swedish temperature data. Part 1: homogenity test for linear trends. International Journal of Climatology, v.17, p.25-34, 1997.

ARTAXO, P.; SILVA DIAS, M.A.F.da; ANDREAE, M.O. O mecanismo da floresta para fazer chover. Scientific American, n.11, p.38-45, 2003.

BENGTSSON, L. Uncertainties of global climate predictions. In: Ed.:Schulze, E.D.; Heimann, M.; Harrison, S.; Holland, E.; Lloyd, J.; Prentice, I.C.; Schimel, D. (Eds.). Global biogeochemical cycles in the climate system. San Diego: Academic Press, 2001. p.15-30.

BONSAL, B.R. et al. Characteristics of daily extreme temperatures over Canada. Journal of Climate, v.14, p.1959-1976, 2001. CHUNG, Y.S.; YOON, M.B. Interpretation of recent temperature and precipitation trends observed in Korea. Theoretical and Applied Climatology, v.67, p.171-180, 2000. 
EASTERLING, D.R. et al. Maximum and minimum temperature trends for the Globe. Science, v.277, p.364-367, 1997.

ESHEL, G. Forecasting the North Atlantic Oscillation using North Pacific Surface Pressure. Monthly Weather Review, v.131, p.1018-1025, 2003.

FLANNERY, T. Os Senhores do clima. Rio de Janeiro, RJ: Record, 2007. 388p.

IPCC. Climate change 2001: synthesis report. Contribution of Working Group I and III to the Third Assessment of the Intergovernmental Panel on Climate Change (IPCC). Cambridge University Press, Cambridge. 2001

KAISER, D.P.; QIAN, Y. Decreasing trends in sunshine duration over China for 1954-1998: Indication of increased haze pollution? Geophysical Research Letters, v.29, n.21, p.2042, 2002.

MARENGO, J.A. Mudanças climáticas globais e seus efeitos na biodiversidade: caracterização do clima atual e definição das alterações climáticas para o território brasileiro ao longo do século XXI. Brasília, DF: MMA, 2006. 212p.
SNEYERS, R. Sur L' analyse statistique des séries d' observations. Genève: Organization Météorologique Mondial, 1975. 192p. (OMM Note Technique, 143).

TEIXEIRA, R.F.B. A Oscilação do Atlântico Norte e a qualidade da quadra chuvosa do Ceará. In: CONGRESSO BRASILEIRO DE METEOROLOGIA, 12, 2002, Foz do Iguaçú. Anais... Foz do Iguaçú, PR: SBMet, 2002. CDRom.

TONKAZ, T.; CETIN, M.; TÜLÜCÜ, K. The impact of water resources development projects on water vapor pressure trends in a semi-arid region, Turkey. Climatic Change, v.82, p.195-209, 2007.

ZHANG, Y.L.; QIN, B.Q.; CHEN, W.M. Analysis of 40 years records of solar radiation data in Shanghai, Nanjing and Hangzhou in Eastern China. Theoretical and Applied Climatology, v.78, p.217-227, 2004.

WILLET, K.M. et al. Attribution of observed surface humidity changes to human influence. Nature, v.449, n.7163, p.710712, 2007. 